\title{
Two-dimensional DOA Estimation in Monostatic MIMO Radar with Double Parallel Uniform Linear Arrays using Propagator Method
}

\author{
Xia Lili1, Zhang Xiaofei2, *, Qiu Xiaofeng 3 \\ 1The PLA Military Representatives Office in Nanjing Daqiao Machine \\ Factory, Nanjing 210000, China \\ 2Department of Electronic Engineering, Nanjing University of \\ aeronautics \& astronautics, Nanjing, 210016, China \\ 3Institute of command information system, PLA University of science and \\ technology, Nanjing, 210007, China \\ *email:fei_zxf@163.com
}

\begin{abstract}
In this paper, we propose a low complexity two-dimensional direction of arrival (2D-DOA) estimation in monostatic multiple-input multiple-output (MIMO) radar with double parallel uniform linear arrays. The proposed algorithm uses propagator method (PM), which does not require any eigen-value decomposition of the covariance matrix and singular value decomposition of the received data, obtains closed-form solution of 2D-DOA, and has very close angle estimation performance to estimation of signal parameters via rotational invariance techniques (ESPRIT) algorithm. Our algorithm can estimate azimuth and elevation angles without additional pair matching in monostatic MIMO radar. Simulation results verify the usefulness of our algorithm.

Keywords: double parallel uniform linear array, DOA estimation, monostatic MIMO radar, propagator method.

\section{Introduction}

Since multiple-input-multiple-output (MIMO) radars use multiple antennas to simultaneously transmit diverse waveforms and utilize multiple antennas to receive the reflected signals, they have many potential advantages over conventional phased-array radars. The direction of arrival (DOA) estimation algorithms in MIMO radar have been investigated in [1-4], and they contain, estimation of signal parameters via rotational invariance techniques (ESPRIT) algorithm, multiple signal classification (MUSIC) algorithm, and Capon algorithm. It is well known that computational complexity of the propagator method (PM) is significantly smaller because the PM does not require any EVD of the covariance matrix, SVD of the received data, or spectrum search [4].Ref.[5]
\end{abstract}


studies DOD and DOA estimation for bistatic MIMO radar with uniform linear array using propagator method, while our work is to estimate 2D-DOA for monistatic MIMO radar with double parallel uniform linear array.

\section{Data Model}

We consider a monostatic MIMO radar system equipped with both double parallel uniform linear arrays for the transmit and receive arrays, in which $2 \mathrm{M}$ elements and $2 \mathrm{~N}$ elements are arranged with half-wavelength spacing between adjacent antennas, respectively. Array antenna structure is shown in Fig.1. We assume that there are $\mathrm{K}$ uncorrelated targets impinging on the antenna array, and the output of the matched filters at the receiver can be expressed as [5]

$\mathbf{x}(t)=\left[\mathbf{a}_{r}\left(\theta_{1}, \phi_{1}\right) \otimes \mathbf{a}_{t}\left(\theta_{1}, \phi_{1}\right), \mathbf{a}_{r}\left(\theta_{2}, \phi_{2}\right) \otimes \mathbf{a}_{t}\left(\theta_{2}, \phi_{2}\right), \cdots\right.$,

$\left.\mathbf{a}_{r}\left(\theta_{K}, \phi_{K}\right) \otimes \mathbf{a}_{t}\left(\theta_{K}, \phi_{K}\right)\right] \mathbf{s}(t)+\mathbf{n}(t)$

where $\theta_{k}, \phi_{k}$ are the elevation and azimuth angle of the kth target, respectively; $\quad \mathbf{s}(t)=\left[s_{1}(t), s_{2}(t), \cdots, s_{K}(t)\right]^{T}, \quad s_{k}(t)=\beta_{k} e^{j 2 \pi f_{k} t} \quad$ with $\quad f_{k} \quad$ being Doppler frequency and $\beta_{k}$ the amplitude. $n(t)$ is a $4 \mathrm{MN} \times 1$ Gaussian white noise vector of zeros mean and covariance matrix $\sigma^{2} \mathbf{I}_{4 M N}$, where $\sigma^{2}$ is the power of the noise. $\mathbf{a}_{r}\left(\theta_{k}, \phi_{k}\right) \otimes \mathbf{a}_{t}\left(\theta_{k}, \phi_{k}\right)$ is the Kronecker product of the receive and the transmit steering vectors for the kth target. We define $\mathbf{A}=\left[\mathbf{a}_{r}\left(\theta_{1}, \phi_{1}\right) \otimes \mathbf{a}_{t}\left(\theta_{1}, \phi_{1}\right), \cdots, \mathbf{a}_{r}\left(\theta_{K}, \phi_{K}\right) \otimes \mathbf{a}_{t}\left(\theta_{K}, \phi_{K}\right)\right]$, and the matrix

A can be denoted by

(2)

$$
\mathbf{A}=\mathbf{A}_{R} \circ \mathbf{A}_{T}=\left[\begin{array}{c}
\mathbf{A}_{R 1} \\
\mathbf{A}_{R 2}
\end{array}\right] \circ \mathbf{A}_{T}=\left[\begin{array}{c}
\mathbf{A}_{R 1} \circ \mathbf{A}_{T} \\
\mathbf{A}_{R 2} \circ \mathbf{A}_{T}
\end{array}\right]=\left[\begin{array}{c}
\mathbf{A}_{T} \\
\mathbf{A}_{T} \boldsymbol{\Phi}_{y} \\
\vdots \\
\mathbf{A}_{T} \dot{\boldsymbol{\Phi}}_{y}{ }^{N-1} \\
\mathbf{A}_{T} \boldsymbol{\Phi}_{x} \\
\mathbf{A}_{T} \boldsymbol{\Phi}_{y} \boldsymbol{\Phi}_{x} \\
\vdots \\
\mathbf{A}_{T}{ }^{N-1}{ }^{N-1} \boldsymbol{\Phi}_{x}
\end{array}\right]
$$

where

$$
\mathbf{A}_{T}=\left[\mathbf{a}_{t}\left(\theta_{1}, \phi_{1}\right), \mathbf{a}_{t}\left(\theta_{2}, \phi_{2}\right), \cdots, \mathbf{a}_{t}\left(\theta_{K}, \phi_{K}\right)\right] \in \square^{2 M \times K}
$$

and $\mathbf{A}_{R}=\left[\mathbf{a}_{r}\left(\theta_{1}, \phi_{1}\right), \mathbf{a}_{r}\left(\theta_{2}, \phi_{2}\right), \cdots, \mathbf{a}_{r}\left(\theta_{K}, \phi_{K}\right)\right] \in \square^{2 N \times K}$ are the transmit direction matrix and the receive direction matrix, respectively. $\mathbf{A}_{R} \circ \mathbf{A}_{T}$ is Khatri-Rao product. $\quad \mathbf{A}_{R 1}$ and $\mathbf{A}_{R 2}$ are direction matrices of the two subarrays in the receive array, and $\quad \mathbf{A}_{R}=\left[\mathbf{A}_{R 1}^{T}, \mathbf{A}_{R 2}^{T}\right]^{T}$

$\boldsymbol{\Phi}_{y}=\operatorname{diag}\left(\exp \left(-j \pi \sin \theta_{1} \sin \phi_{1}\right), \exp \left(-j \pi \sin \theta_{2} \sin \phi_{2}\right), \cdots, \exp \left(-j \pi \sin \theta_{K} \sin \phi_{K}\right)\right)$ 
$\boldsymbol{\Phi}_{x}=\operatorname{diag}\left(\exp \left(-j \pi \sin \theta_{1} \cos \phi_{1}\right), \exp \left(-j \pi \sin \theta_{2} \cos \phi_{2}\right), \cdots, \exp \left(-j \pi \sin \theta_{K} \cos \phi_{K}\right)\right)$

There exists a $4 \mathrm{MN} \times 4 \mathrm{MN}$ transformation matrix $\mathbf{T}$ corresponding to the finite number of row interchanged operations such that

$$
\mathbf{T A}=\left[\begin{array}{c}
\mathbf{A}_{T} \\
\mathbf{A}_{T} \boldsymbol{\Phi}_{x} \\
\mathbf{A}_{T} \boldsymbol{\Phi}_{y} \\
\mathbf{A}_{T} \boldsymbol{\Phi}_{x} \boldsymbol{\Phi}_{y} \\
\vdots \\
\mathbf{A}_{T} \boldsymbol{\Phi}_{y}{ }^{N-1} \\
\mathbf{A}_{T} \boldsymbol{\Phi}_{x} \boldsymbol{\Phi}_{y}{ }^{N-1}
\end{array}\right]
$$

For the signal model in (1), the covariance matrix $\mathbf{R}_{x}=E\left\{\mathbf{x}(t) \mathbf{x}^{H}(t)\right\}$ can be estimated with L snapshots by $\hat{\mathbf{R}}_{x}=\frac{1}{L} \sum_{l=1}^{L} \mathbf{x}\left(t_{l}\right) \mathbf{x}^{H}\left(t_{l}\right)$.

\section{Angle Estimation in MIMO-radar}

The matrix A can be denoted by

$$
\mathbf{A}=\left[\begin{array}{l}
\mathbf{A}_{1} \\
\mathbf{A}_{2}
\end{array}\right]
$$

where $\mathbf{A}_{1} \in \square^{K \times K}$ is the full-rank matrix, $\mathbf{A}_{2} \in \square^{(4 M N-K) \times K}$. The propagator is a unique linear operator which can be written as [4], $\quad \mathbf{A}_{2}=\mathbf{P}_{c} \mathbf{A}_{1}$, where $\mathbf{P}_{c}$ is the propagator matrix. We define the matrix $\mathbf{P} \in \square^{4 M N \times K}$

$$
\mathbf{P}=\left[\begin{array}{l}
\mathbf{I}_{K} \\
\mathbf{P}_{c}
\end{array}\right]
$$

According to Eqs.(4-5), in the noiseless case, we have

$$
\mathbf{P A}_{1}=\left[\begin{array}{l}
\mathbf{A}_{1} \\
\mathbf{A}_{2}
\end{array}\right]=\mathbf{A}
$$

We can partition the matrix $\mathbf{P}$ as follows

$$
\mathbf{P}=\left[\begin{array}{l}
\mathbf{P}_{1} \\
\mathbf{P}_{2}
\end{array}\right]
$$

where $\mathbf{P}_{n} \in \square^{2 M N \times K}$, for $\mathrm{n}=1,2$. And we have 


$$
\mathbf{P A}_{1}=\left[\begin{array}{c}
\mathbf{P}_{1} \\
\mathbf{P}_{2}
\end{array}\right] \mathbf{A}_{1}=\mathbf{A}=\left[\begin{array}{c}
\mathbf{A}_{T} \\
\mathbf{A}_{T} \boldsymbol{\Phi}_{y} \\
\vdots \\
\mathbf{A}_{T} \dot{\boldsymbol{\Phi}}_{y}{ }^{N-1} \\
\mathbf{A}_{T} \boldsymbol{\Phi}_{x} \\
\mathbf{A}_{T} \boldsymbol{\Phi}_{y} \boldsymbol{\Phi}_{x} \\
\vdots \\
\mathbf{A}_{T} \boldsymbol{\Phi}_{y}{ }^{N-1} \boldsymbol{\Phi}_{x}
\end{array}\right]
$$

According to Eq. (8), $\mathbf{P}_{2} \mathbf{A}_{1}=\mathbf{P}_{1} \mathbf{A}_{1} \boldsymbol{\Phi}_{x}$, and we can write $\mathbf{P}_{1}^{+} \mathbf{P}_{2}=\mathbf{A}_{1} \boldsymbol{\Phi}_{x} \mathbf{A}_{1}^{-1}$. Define $\boldsymbol{\Psi}_{x}=\mathbf{P}_{1}^{+} \mathbf{P}_{2}$. Because $\boldsymbol{\Psi}_{x}$ has the same eigenvalues as $\boldsymbol{\Phi}_{x}$, we use EVD of $\boldsymbol{\Psi}_{x}$ to get $\boldsymbol{\Phi}_{x}^{\prime}$. Also we obtain an estimation $\mathbf{A}_{1}^{\prime}$ via the eigen vectors of $\boldsymbol{\Psi}_{x}$. In no-noise case, $\boldsymbol{\Phi}_{x}^{\prime}=\Pi^{T} \boldsymbol{\Phi}_{x} \Pi, \mathbf{A}_{1}^{\prime}=\mathbf{A}_{1} \Pi$, where $\Pi$ is a permutation matrix, which satisfies $\boldsymbol{\Pi}^{-1}=\boldsymbol{\Pi}^{T}$. And then we have the estimation $\mathbf{A}^{\prime}$ of the matrix $\mathbf{A}, \mathbf{A}^{\prime}=\mathbf{P} \mathbf{A}_{1}^{\prime}=\mathbf{A} \Delta \Pi$, where $\Delta$ is the diagonal scaling matrix. And we construct the following matrix

(9)

$$
\mathbf{A}^{\prime \prime}=\mathbf{T} \mathbf{A}^{\prime}=\mathbf{T} \mathbf{P} \mathbf{A}_{1}^{\prime}=\mathbf{T} \mathbf{A} \Delta \Pi=\left[\begin{array}{c}
\mathbf{A}_{T} \\
\mathbf{A}_{T} \boldsymbol{\Phi}_{x} \\
\mathbf{A}_{T} \boldsymbol{\Phi}_{y} \\
\mathbf{A}_{T} \boldsymbol{\Phi}_{x} \boldsymbol{\Phi}_{y} \\
\vdots \\
\mathbf{A}_{T} \boldsymbol{\Phi}_{y}{ }^{N-1} \\
\mathbf{A}_{T} \mathbf{\Phi}_{x} \boldsymbol{\Phi}_{y}{ }^{N-1}
\end{array}\right] \Pi
$$

We partition the matrix $\mathbf{A}^{\prime \prime}$ as $\mathbf{A}^{\prime \prime}=\left[\begin{array}{lll}\mathbf{A}_{1}^{\prime \prime T} & \mathbf{A}_{2}^{\prime \prime T} & \mathbf{A}_{N}^{\prime \prime T}\end{array}\right]^{T}$, where $\mathbf{A}_{n}^{\prime} \in \square^{4 M \times K} \quad$, for $\mathrm{n}=1, \cdots, \mathrm{N}$. Define $\mathbf{A}_{a}^{\prime \prime}=\left[\mathbf{A}_{1}^{\prime \prime T}, \mathbf{A}_{2}^{\prime \prime T}, \cdots, \mathbf{A}_{N-1}^{\prime \prime T}\right]^{T}$ and $\mathbf{A}_{b}^{\prime \prime}=\left[\mathbf{A}_{2}^{\prime \prime}, \mathbf{A}_{3}^{\prime \prime T}, \cdots, \mathbf{A}_{N}^{\prime^{T}}\right]^{T}$. In no- noise case, $\mathbf{A}_{b}^{\prime \prime}=\mathbf{A}_{a}^{\prime \prime} \Pi^{T} \mathbf{\Phi}_{y} \Pi$, we define $\boldsymbol{\Phi}_{y}^{\prime}=\Pi^{T} \boldsymbol{\Phi}_{y} \Pi$ 。 In no-noise case, we obtain $\boldsymbol{\Phi}_{y}^{\prime}$ estimation via $\mathbf{\Phi}_{y}^{\prime}=\left[\mathbf{A}_{a}^{\prime}\right]^{+} \mathbf{A}_{b}^{\prime}$ 。

The elevation angle $\theta_{k}$ and azimuth angle $\phi_{k}$ are estimated via

$$
\begin{gathered}
\hat{\theta}_{k}=\sin ^{-1} \sqrt{\left.\left[j \llbracket \ln \left(\operatorname{diag}^{-1}\left[\hat{\mathbf{\Phi}}_{y}\right]\right) / \pi\right]^{2}+\left[j \square \ln \left(\operatorname{diag}^{-1}\left[\hat{\mathbf{\Phi}}_{x}\right]\right) / \pi\right)\right]^{2}}, \\
\hat{\phi}_{k}=\tan ^{-1}\left(\frac{\ln \left(\operatorname{diag}^{-1}\left[\hat{\boldsymbol{\Phi}}_{y}\right]\right)}{\ln \left(\operatorname{diag}^{-1}\left[\hat{\boldsymbol{\Phi}}_{x}\right]\right)}\right),
\end{gathered}
$$

where $\operatorname{diag}^{-1}[\mathbf{A}]$ means a vector with the diagonal elements of the matrix $\mathbf{A}$, $\ln ($.$) stands for natural logarithm.$ 
We partition the covariance matrix $\hat{\mathbf{R}}_{x}=\left[\hat{\mathbf{R}}_{1}, \hat{\mathbf{R}}_{2}\right]$, where $\hat{\mathbf{R}}_{1} \in \square^{4 M N \times K}$, $\hat{\mathbf{R}}_{2} \in \square^{4 M N \times(4 M N-K)}$. We can get the estimate of the matrix $\mathbf{P}_{c}$ via $\hat{\mathbf{P}}_{c}=\left[\hat{\mathbf{R}}_{1}^{+} \hat{\mathbf{R}}_{2}\right]^{T}$. In contrast to ESPRIT algorithm, our algorithm has a low computational load because the PM does not require any EVD of the covariance matrix.

\section{Simulation Results}

We assume that there are $\mathrm{K}=3$ non-coherent targets, which are located at angles $\left(\theta_{1}, \phi_{1}\right)=\left(10^{\circ}, 15^{\circ}\right),\left(\theta_{2}, \phi_{2}\right)=\left(20^{\circ}, 25^{\circ}\right) \quad$ and $\left(\theta_{3}, \phi_{3}\right)=\left(30^{\circ}, 35^{\circ}\right)$, respectively. Fig.2 presents angle estimation result of our algorithm for all three targets with $\mathrm{M}=8, \mathrm{~N}=8, \mathrm{~L}=100 \mathrm{SNR}=4 \mathrm{~dB}$ and $12 \mathrm{~dB}$, respectively. From Fig. 2, azimuth angle and elevation angle can be clearly observed. Fig.3 shows the angle estimation performance comparison with $\mathrm{M}=8, \mathrm{~N}=8$ and $\mathrm{L}=100$, where we compare our algorithm with ESPRIT algorithm and CRB. It is indicated in Fig.6 that our algorithm has very close angle estimation performance to ESPRIT algorithm. Fig. 4 presents angle estimation performance with two closely spaced targets, where $\mathrm{M}=8, \mathrm{~N}=8$ and $\mathrm{L}=100$ are considered. The two closely spaced targets are located at $\left(\theta_{1}, \phi_{1}\right)=\left(10^{\circ}, 15^{\circ}\right),\left(\theta_{2}, \phi_{2}\right)=\left(13^{\circ}, 18^{\circ}\right)$ From Fig.4, we find our algorithm works well in the case of closely spaced targets.

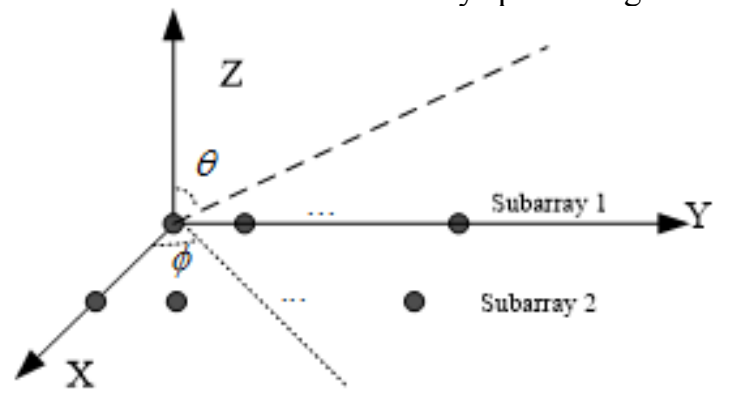




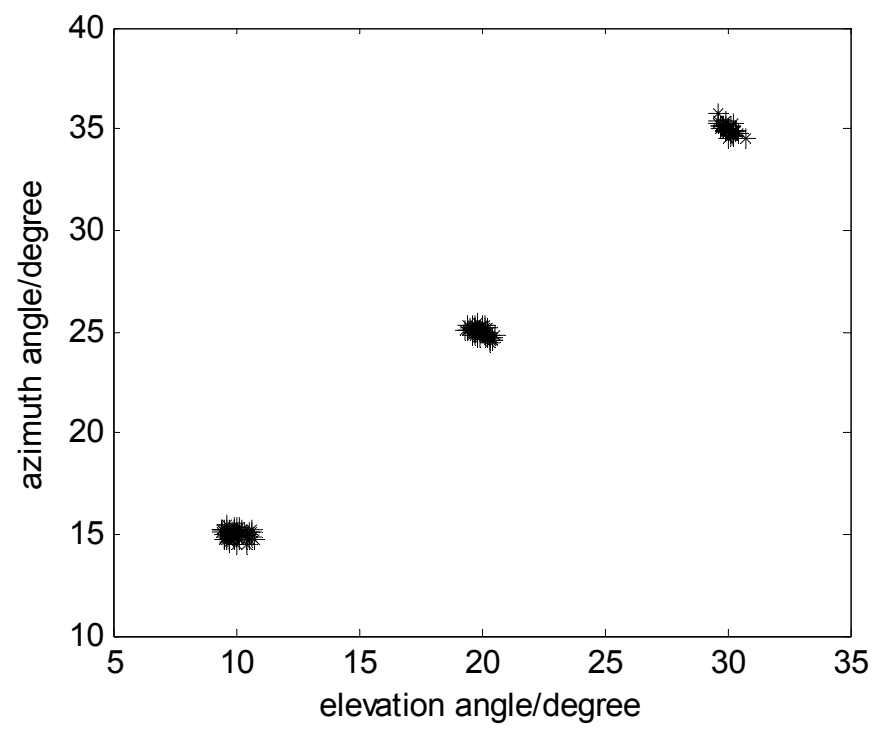

Fig.1 Array antenna structure Fig.2 Angle estimation results with $4 \mathrm{~dB}$

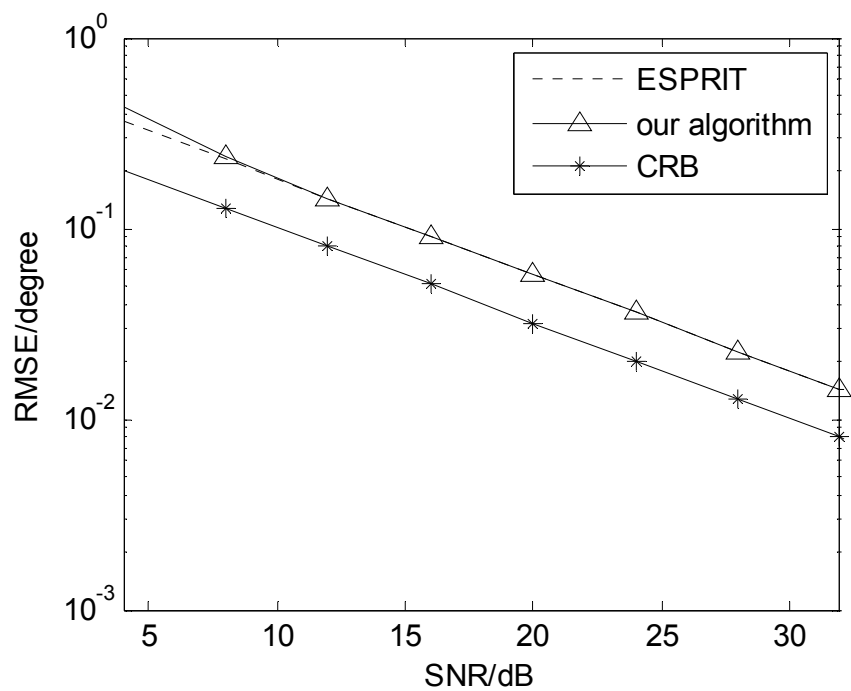




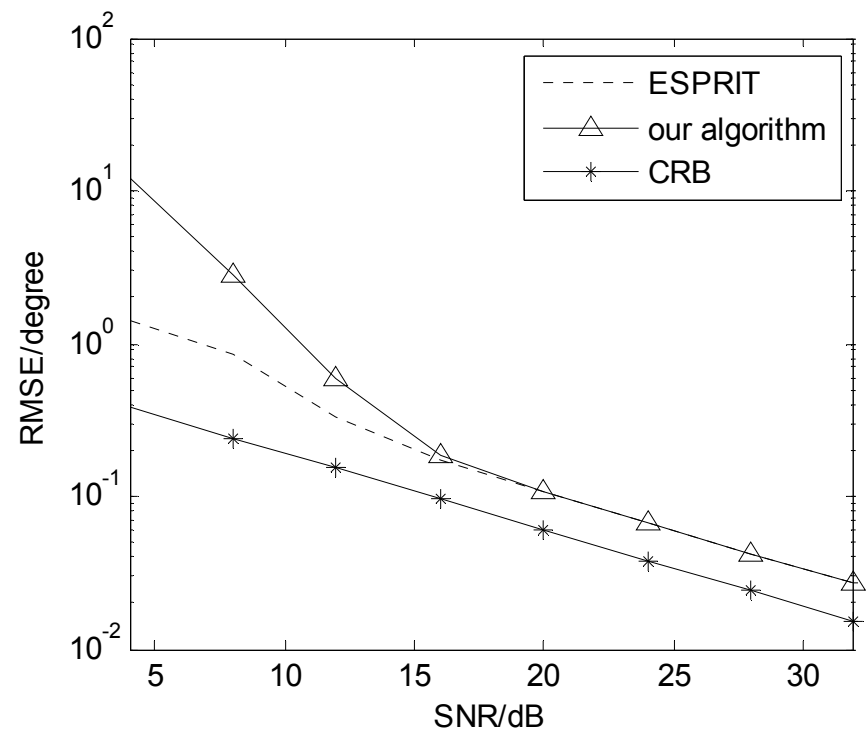

Fig.3 Angle estimation comparison Fig. 4 Angle estimation with two closely spaced targets

\section{Conclusion}

In this paper, we have proposed a low-complexity two-dimensional angle estimation for the monostatic MIMO radar with double parallel uniform linear arrays using PM algorithm. The proposed algorithm obtains closed-form solution of 2D-DOA, has very close angle estimation performance to ESPRIT, and then the complexity load of our algorithm is less than that of ESPRIT.

\section{References}

[1] C. Duofang, C. Baixiao, and Q. Guodong, "Angle estimation using ESPRIT in MIMO radar,” Electron. Lett., vol. 44, no. 12, pp. 770-771, 2008.

[2] X. Zhang, D. Xu. "Angle estimation in MIMO radar using reduced-dimension Capon,” Electronic letter, 2010, 46 (12): 860 - 861.

[3] X. Zhang, L. Xu, L. Xu, et al. "Direction of Departure (DOD) and Direction of Arrival (DOA) Estimation in MIMO radar with Reduced-dimension MUSIC," IEEE Communications Letters, 2010, vol.14 no.12, pp.1161 - 1163, 2010 .

[4] Sylvie Marcos, Alain Marsal and Messaoud Benidir.The propagator method for source bearing estimation, Signal Processing, vol. 42, no. 2, pp. 121-138, 
March 1995.

[5] Zhang xiaofei,Xu dazhuan, Computationally efficient DOD and DOA estimation for bistatic MIMO radar with propagator method, International Journal of Electronics, vol. 99, no. 9, pp. 1207-1221, 2012 